\title{
Lightning Currents on Fastening Assemblies of an Aircraft Fuel Tank, Part III: Validation of the Current Assessment Method with Measurement
}

\author{
Paul Monferran, Christophe Guiffaut, Member, IEEE, Alain Reineix, Member, IEEE Fabian Fustin, and Fabrice \\ Tristant
}

\begin{abstract}
This paper proposes a validation of the lightning current assessment method in the aircraft assemblies proposed in the previous parts. In particular, it concerns the FDTD fastener modeling for which the variability of its primary parameter has to be taken into account. The principle is to merge an electromagnetic fastener model with an uncertainty model. The fastener model proposed in this paper is an improvement of the model of part I; a wire with a lumped resistor on a low conductive plate. This model is able to ensure a good shielding efficiency without current distribution disruptions provided that the conductivity of the plate is weak in contrast to the lumped resistor. This model has the benefits to be able to represent any fastener without location constraint in the FDTD grid. A log-normal distribution has been previously established as uncertainty model which represents the variability of the resistive parameter. The fastener model is included in the FDTD modeling of a realistic fuel tank with each lumped resistor taking a probabilistic value according to the log-normal law. To take into account the variability of resistors, several simulations with different sets of resistance values are performed. The aim is to surround current measurements with simulated currents at various location of the fuel tank. Lightning current is directly injected on a fastener in simulation as in measurement. This paper demonstrates that a specific attention must be given to the modeling of this fastener for the current assessment close to the lightning injection. Moreover, the results highlight the relevance of the use of an uncertainty model and its reliability for the lightning current assessment.
\end{abstract}

Index Terms-uncertainties model, fastener model, finitedifference time domain (FDTD), lightning issue, aircraft fuel tank.

\section{INTRODUCTION}

A $\mathrm{N}$ accurate lightning current estimation on an aircraft fuel tank is not practicable. In particular, the fastener modeling is difficult. In the literature, all the proposed fastener models [1]-[4] have the same lack: the main parameter of the model cannot be accurately identified. This parameter is a resistive parameter which represents the contact resistances. All the previous models use nominal values around few milliohms. The inherent inaccuracy of their results is due to strong uncertainties about the contact resistances values. Indeed, it

The writing of this paper was supported by the XLIM Laboratory.

P. Monferran, C. Guiffaut and A. Reineix are with Xlim laboratory, 123 avenue Albert Thomas, 87000 Limoges, France (phone: +335-55-45-77-39; fax: +33-5-55-45-77-66; e-mail : paul.monferran@xlim.fr, christophe.guiffaut@xlim.fr and alain.reineix@xlim.fr)

F. Fustin and F. Tristant are with Dassault Aviation Company, 78 Quai Marcel Dassault, 92552 Saint-Cloud, France (e-mail: fabian.fustin@dassaultaviation.com and fabrice.tristant@dassault-aviation.com) has been demonstrated that their variability is strong and has an important impact for the lightning current estimation [3], [5], [6].

In part II of this three-part paper [7], a method dealing with DC measurement and an optimization process is proposed and numerically validated for the characterization of contact resistances. Nevertheless, this method is sensitive with measurement errors. Moreover, the characterization method is not a conceivable solution for complex structures with a high number of fasteners.

The proposed approach in [6], [7] is to merge an uncertainties model with a FDTD fastener model. A general method applied to the contact resistance uncertainties allows defining a probability law as uncertainties model [6]. This model represents the variability of the equivalent fastener resistance whatever the fastener type. It has been defined from a measurement database with different types of fastener with and without default. We note that the proposed model depicts the contact resistance variation for fastener after lightning shot. In practice, the resistive parameter of fastener model has a probabilistic value according to the uncertainties model. In part I, the fastener model is a wire with a resistance (SM). Therefore, the resistance takes a probabilistic value conforming to the log-normal law defined in part I. For complex fastener model composed by a resistance network as in part II (CM), each resistance of network follows another law. This law is defined provided that 1) each resistance follows the same law and 2) the use of a specific bounding procedure on the log-normal law [7]. In the first two parts, the models are implemented on a generic fuel tank modeling (see Fig. I- $10^{1}$ ). In order to take into account the uncertainties, several simulations with different probabilistic sets of resistance are made. The results highlight the need of the use of an uncertainties model for the lightning current assessment. Furthermore, both fastener modelings require to open the structure. Thus, a question is raised about the relevance of the proposed fastener models concerning the field calculation inside confined structure.

In this paper, the inherent field calculation errors due to the SM model are explored with the same generic fuel tank as in the previous parts. This model can involve an important electrical field (E-field) penetration. Hence, a new FDTD fastener model is proposed. A low conductor electrostatic

\footnotetext{
${ }^{1}$ Henceforth, equations, figures, or sections from Part $X$ in [6], [7] are referred to using the prefix $X \in\{I, I I\}$, e.g., Fig. I-1 or Eq. (II-1).
} 
shield (ES) is added on the made aperture. A compromise between the losses on the ES and the shielding efficiency is studied. Then, measurement are made on a realistic aircraft fuel tank in different locations. The uncertainties model is combined with the new fastener model. As in [6], [7], several simulations are realized in order to surround measurement. In addition, a sensitivity analysis on modeling is carried out in order to enhance the proposed surrounding.

First, the new fastener model which solves the field calculation errors is presented in section II. In section III, the fuel tank measurement and modeling are depicted. Then, the comparison between the measurement and the simulated currents is carried out in section IV.

\section{MOdELING INCIDENCE ON FIELD CALCULATION}

\section{A. Shielding efficiency issue}

The SM presented in part I involves to create apertures in the structure. Indeed, the fasteners are represented with a resistive wire. The link between two assemblies is realized with this wire. Thus, the shielding efficiency can be reconsidered in confined zone as a fuel tank. Fig. 1 represents four E-field mappings which cut the generic fuel tank of Fig. I-10. The fuel tank is perfectly metallic and all the fastening assembly resistances are at $5 \mathrm{~m} \Omega$. The borders (with the higher E-field in red) of the yz map corresponds to the E-field around the fuel tank (external E-field). For the xy map, the external E-field is only represented along the y-axis. Fig. 1 highlights the weak shielding efficiency using the SM as fastener model at 100 Hz. Indeed, the E-field penetration seems to be particularly strong inside the fuel tank and not only confined close to the aperture.

A $1 \mathrm{~mm}$ thickness plate with an equivalent conductivity to the fastening line $\left(\sigma=4 \cdot 10^{5} \mathrm{~S} . \mathrm{m}^{-1}\right)$ is used as fastener model. This kind of model, noted HPM, is already validated in [4] as equivalent to SM in terms of global current distribution. The HPM is employed as a relative reference for the shielding efficiency for the reason that it does not involve aperture in the structure. Fig. 2 illustrates the E-field mapping on the generic fuel tank using the HPM. We note that both fastener models have the same behavior for the external E-field. However, the shielding efficiency is much better with the HPM than the SM by comparing both E-field inside the fuel tank.

\section{B. The hybrid model}

The aim of this new model is to keep the current distribution behavior of the SM while ensuring a good shielding efficiency. The idea is to combine the SM with the HPM adding a low conductive plate on the SM. The conductivity of the added plate has to be insignificant compared to the SM resistance. Thus, this plate is only used as an ES. Fig. 3 presents this hybrid model (SM+HPM). The FDTD representation of the fastening line of the generic fuel tank is illustrated in Fig. 4. A compromise has to be found between the losses on the plate and the shielding efficiency. The thin-plate-model for the ES FDTD modeling [8]-[10].
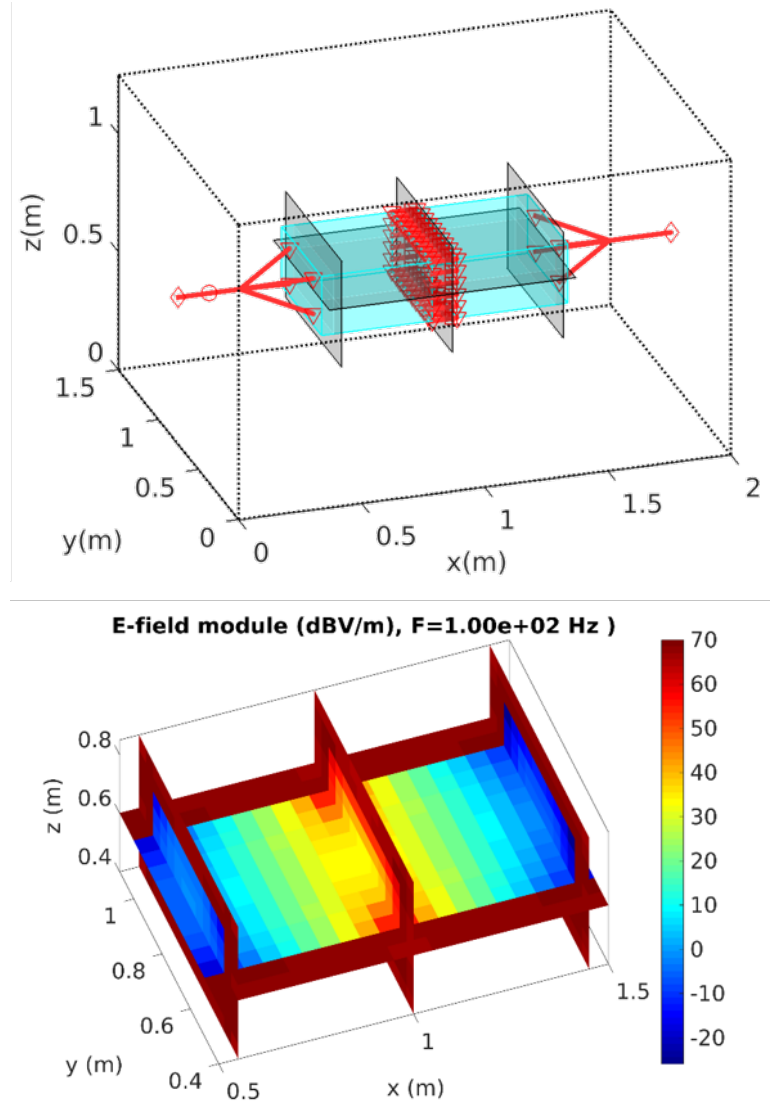

Fig. 1. E-field mapping on the generic fuel tank with the SM as fastener model. At top, the proposed mapping (in black). At bottom, the E-field module calculation at $100 \mathrm{~Hz}$.

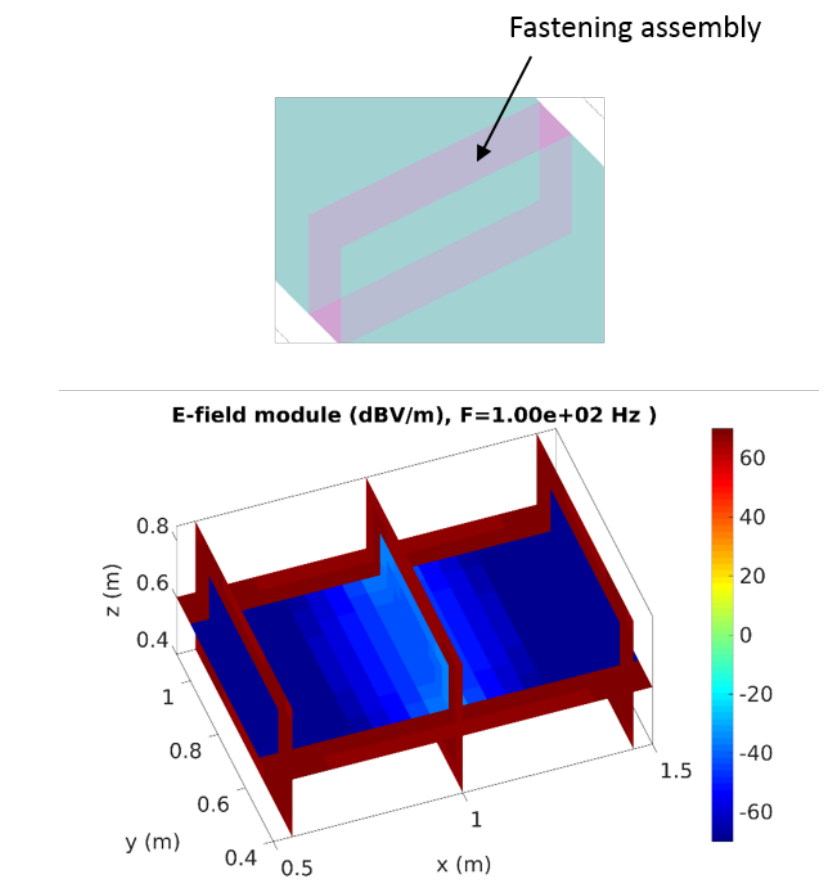

Fig. 2. E-field mapping on the generic fuel tank with the HPM as fastener model. At top, the proposed fastener model. At bottom, the E-field module calculation at $100 \mathrm{~Hz}$. 


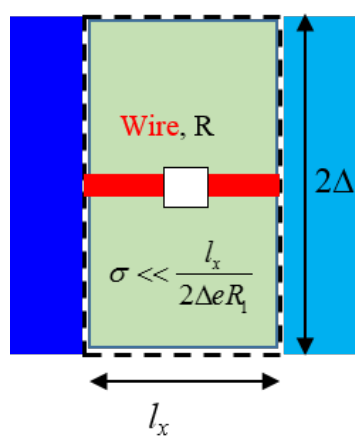

Fig. 3. Hybrid fastener model (SM+HPM).

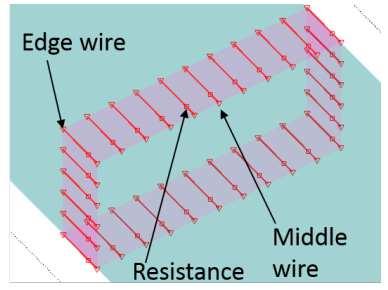

Fig. 4. FDTD representation of the hybrid model.

\section{Shielding efficiency analysis}

The same modeling as Fig. 1 is computed replacing the SM with the hybrid model. As a reminder, the equivalent conductivity of the fastening assembly is $\sigma=4 \cdot 10^{5} \mathrm{~S} . \mathrm{m}^{-1}$ for a $1 \mathrm{~mm}$ thickness plate. Four simulations are performed with four different conductivities $\sigma_{E S}$ associated to the ES of the hybrid model. The mean shielding efficiency is calculated for each field map at several frequencies $f$ using the following equation :

$$
\operatorname{Err}_{\text {mean }}(f)=\frac{1}{n} \sum_{i=1}^{n}\left\|\overrightarrow{E_{\text {HPM }}^{i}(f)}\right\|-\left\|\overrightarrow{E_{\text {model }}^{i}(f)}\right\|
$$

with $n$ the number of calculated E-field points.

This error is also calculated for the magnetic field (H-field) replacing $E$ with $H$ in (1). The results are presented in Fig. 5. First, we note that the hybrid model enhances significantly the shielding efficiency. The $\mathrm{H}$-field is well computed for any models. The relative errors are explained by the intrinsic difference between modelings. On the other hand, the E-field shielding efficiency is ensured with the hybrid model even with a low conductive ES with $400 \mathrm{~S}^{-1} \mathrm{~m}^{-1}$ corresponding to $\sigma_{E S}=\frac{\sigma}{1000}$ in contrast to the SM. The ES conductivity has a weak impact for the shielding efficiency. As expected, the mean error increases with frequency. The computed mean error is important for the E-field at $1 \mathrm{MHz}$ for the hybrid model. Nevertheless, this absolute error is insignificant since the Efield module is weak as shown in Fig. 6. Thus, the shielding efficiency is ensured with the hybrid model in the lightning frequency range.

In order to be consistent, the current losses involved by the ES adding are studied. For this purpose, the currents on the middle and edge wires (cf Fig. 4) are analyzed. We compare the computed currents of the hybrid model to the SM. Fig. 7 depicts the results. It clearly highlights that the ES conductivity $\sigma_{E S}$ has to be at least one hundred times less than the equivalent fastening assembly conductivity $\sigma$. Otherwise, the losses are sufficiently significant. The conductivity $\sigma$ is influenced by the highest resistance value of the fastening assembly. If this value is high, $\sigma$ is small and hence $\sigma_{E S}$ one hundred
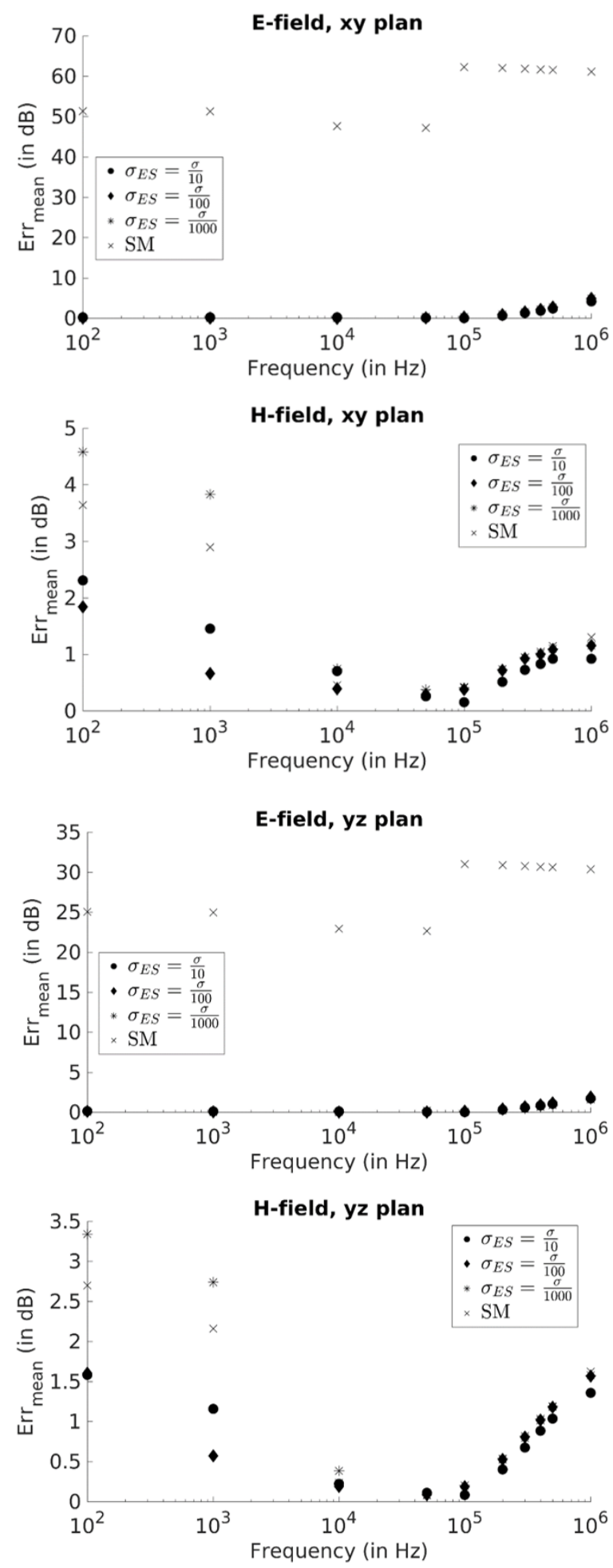

Fig. 5. Mean electric and magnetic field errors calculated using (1) of the SM and the hybrid model with different ES conductivities. 


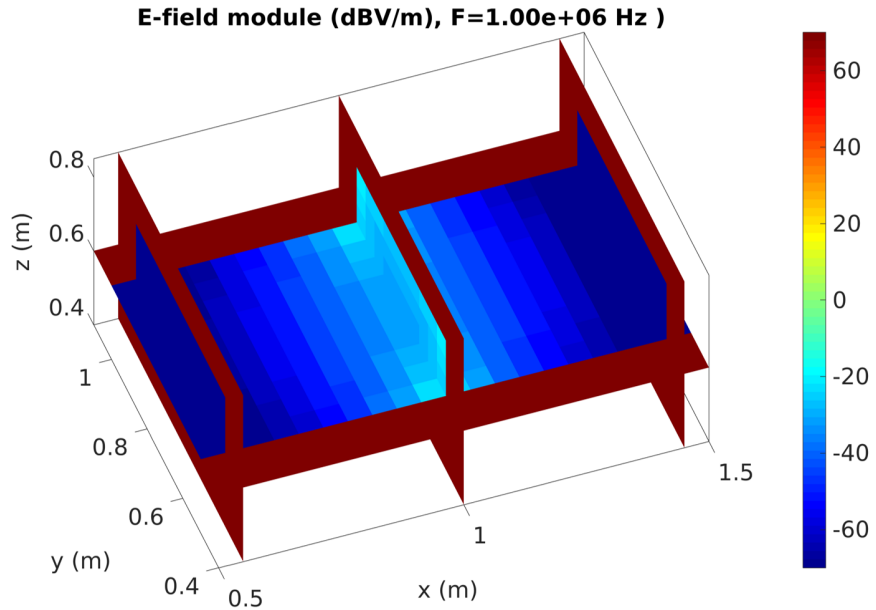

Fig. 6. E-field module calculation at $1 \mathrm{MHz}$ for the hybrid model with $\sigma_{E S}=$ 400 S.m ${ }^{-1}$
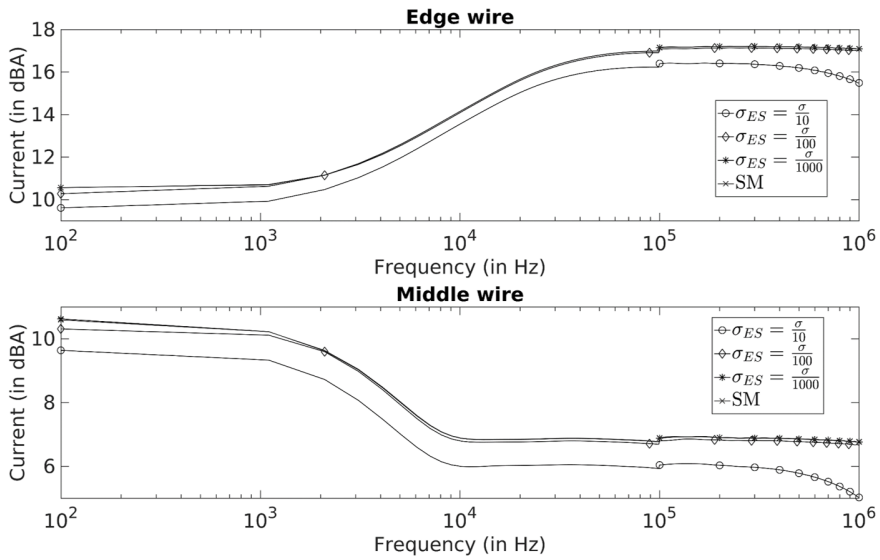

Fig. 7. Current distribution on the edge wire (at top) and middle wire (at bottom) of the fastening assembly in function of frequency for the hybrid model with different ES conductivities and the SM.

times smaller. As a consequence, the shielding efficiency could be questioned. Nevertheless, in our experience, a very low conductive plate is still efficient as electric shield and the fastening resistance values are usually weak (less than 100 $\mathrm{m} \Omega)$.

\section{FUEL TANK MODELING}

\section{A. Measurement bench}

A measurement campaign has been realized by Dassault Aviation on a validation box which represents a fuel tank. This fuel tank under test is presented in Fig. 8. A lightning current is directly injected on a fastener. This configuration is usually called attachment configuration. A zoom on the lightning current injection is shown in Fig. 9. The injected lightning waveform is a double-exponential with $12.3 \mu$ s rise time, $38.4 \mu$ s pulse length and $95.73 \mathrm{kA}$ amplitude. The fuel tank undergoes nine lightning shots with the same lightning injection. The injected currents are measured until $1 \mathrm{~ms}$ as illustrated in Fig. 10. Several current measurements are also realized at various locations during each lightning shot with Pearson and Rogowski probes. In this paper, we focus on the

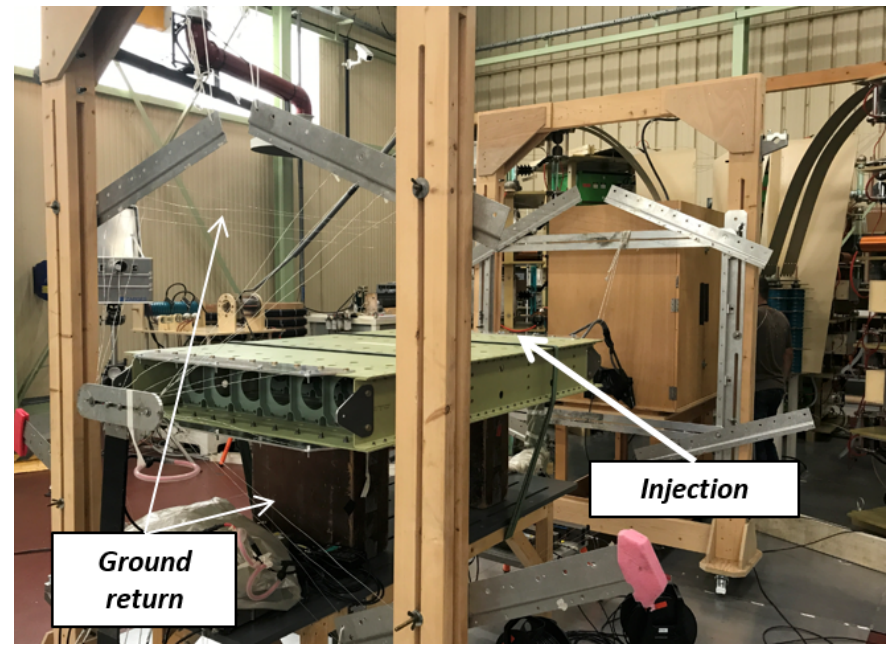

Fig. 8. Photography of the test bench.

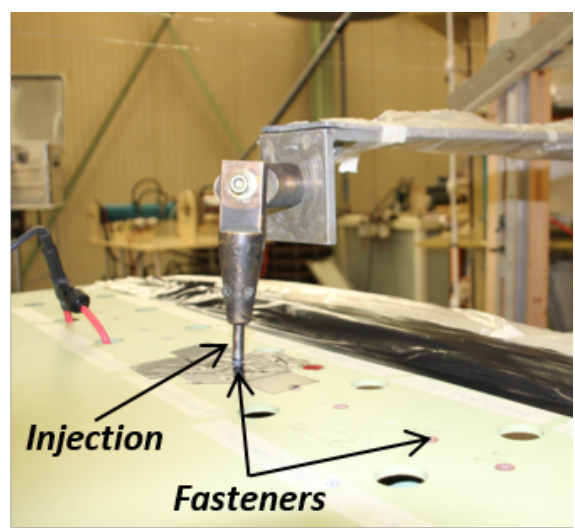

Fig. 9. Photography of the lightning current injection.

current measurements made on the ribs of the fuel tank with a Pearson probe as shown in Fig. 11. The probe is inserted in the two holes around the rib and thus it measures the current flowing in the rib.

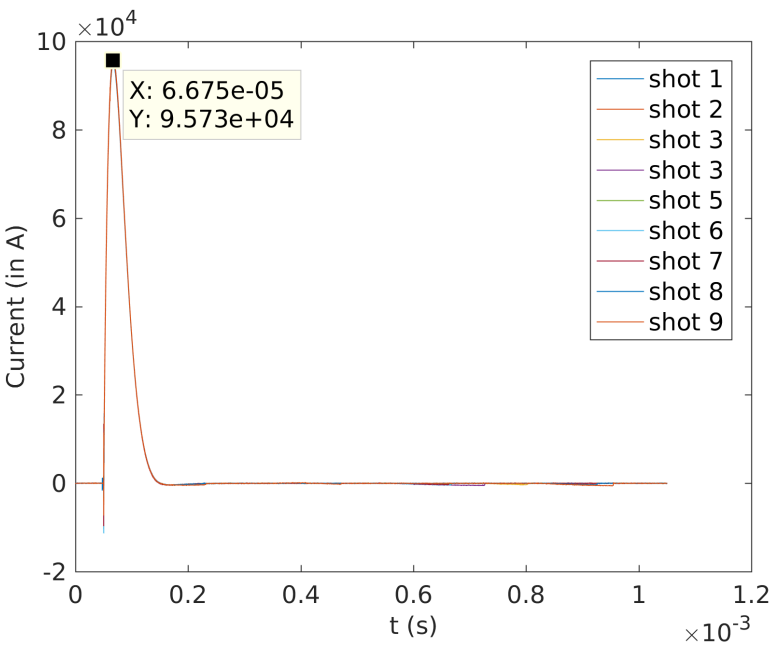

Fig. 10. Measurement of the injected currents. 


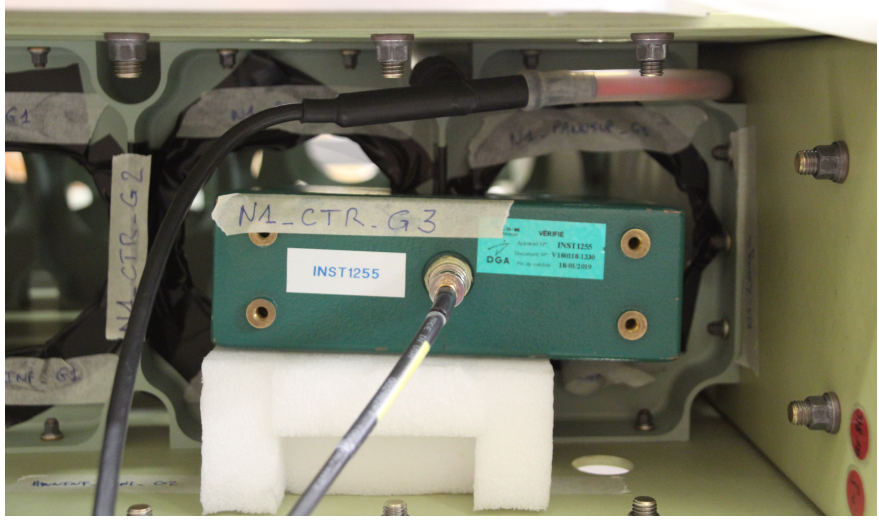

Fig. 11. Photography of the current measurement on rib with a Pearson probe.

\section{B. Fuel tank design}

The dimensions of the fuel tank are $174 \times 117 \times 21 \mathrm{~cm}^{3}$. It is constituted with several elements: ribs, panels, wedges, spars... Fig. 12 depicts the computer-aided design of the fuel tank with all the elements which compose it. These elements are screwed together with fasteners. Overall, $255 \mathrm{RH}$ fasteners (Fig. I-1) are used for the fuel tank assembly. The panels and spars are composite. The wedges are in aluminum as the ribs. Extended copper foils (ECF) are added on the panels along the spars and ribs interfaces. These copper foils (ECF815 and ECF195) are usually used as lightning attachment protection. The ECF815 is a 815 g.m $\mathrm{m}^{-2}$ copper mesh which ensure a better protection as the ECF195 which is a 195 g.m ${ }^{-2}$ copper mesh. The ECF195 is applied in the both panels of the fuel tank in contrast to the ECF815 which only exists on the lightning attachment panel. Moreover, each element is painted with an aircraft paint. Furthermore, the fuel tank is drilled at several locations in order to insert a current measurement probe. Measurement are also realized between two holes of rib.

\section{Fuel tank FDTD modeling}

The test bench is meshed in a cubic grid. The fuel tank with the injection set-up and the return paths are taken into account. Fig. 13 illustrates the FDTD mesh. In order to be consistent with the fuel tank geometry, the mesh step is 1.5 $\mathrm{cm}$. Perfectly matched layers (PML) are used as boundary conditions to absorb the waves [11]-[13]. The size of solution without the PML is $185 \times 152 \times 122$ cells.

The paint is not represented in the FDTD modeling. Some element features used for the modeling are presented in Table I. The surface impedance of this table are calculated from the conductivity measurements of each material presented in Table II. An equivalent conductivity is proposed for the panel/ECF interfaces. Thus, theses interfaces are represented with a homogeneous plate model in the FDTD modeling. Moreover, all the others modeled plates (injection set-up and ground plates) are $2 \mathrm{~mm}$ thickness aluminum plates and hence they have the same surface impedance as the ribs. The low frequency thin-plate-model [8]-[10] is used for FDTD plate
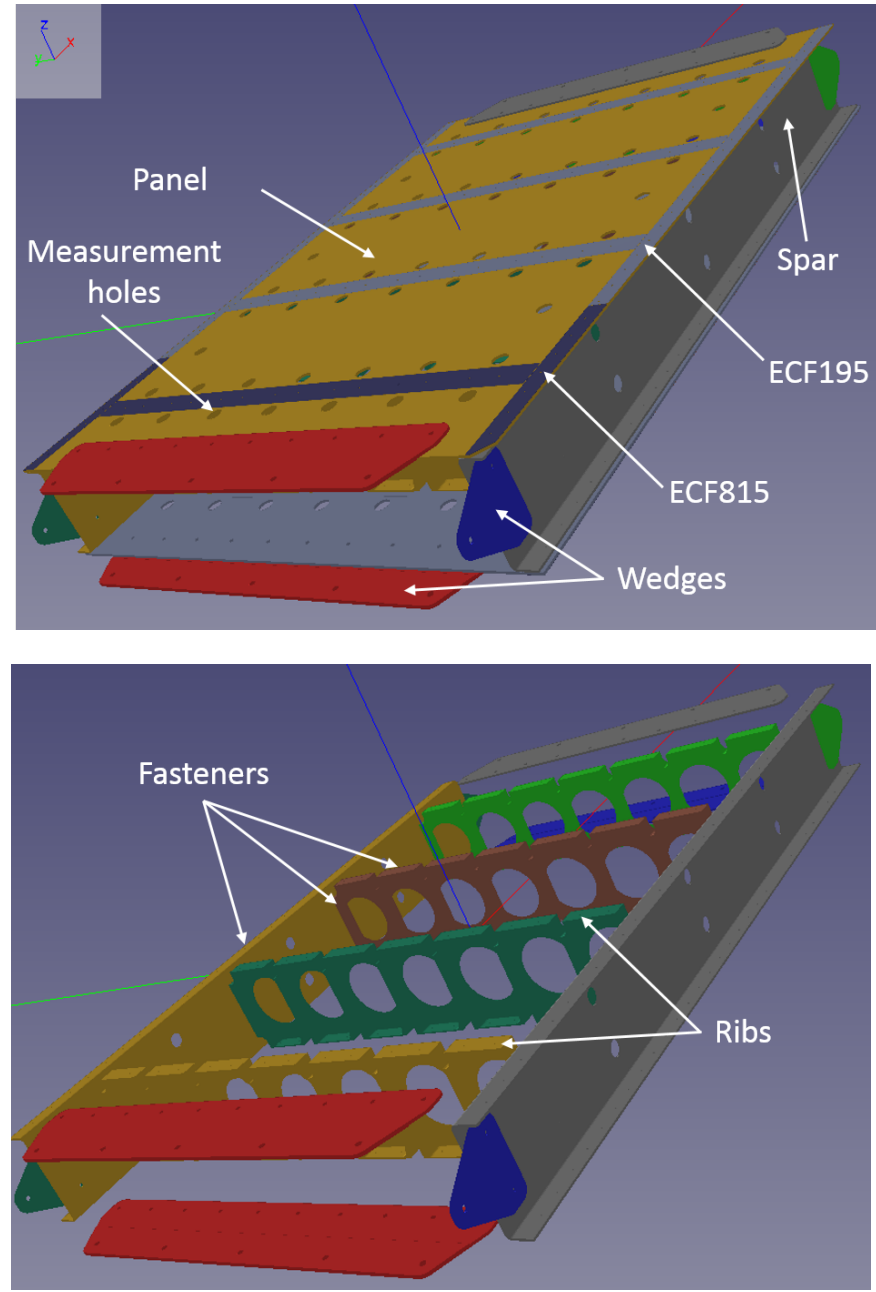

Fig. 12. Fuel tank CAD. At top, full CAD. At bottom, CAD without panels.

modeling. Furthermore, the ground wire are represented with a $4.5 \mathrm{~mm}$ radius and a $0.26 \mathrm{~m} \Omega \mathrm{m}^{-1}$ resistance per unit length. In addition, the junctions between the ground wires and the plates are modeled with a $5 \mathrm{~m} \Omega$ lumped resistance.

At each interface (panel/rib, spar/panel, etc...), the current can flow only through fasteners. The hybrid fastener model of section II is used. Fig. 14 presents the implementation of the hybrid fastener model. As in part I and part II, the fastener wire radius size is $1 / 3$ of the mesh step, i.e $0.5 \mathrm{~mm}$ for this modeling. Each lumped resistance of the fastener model takes a probabilistic random value according to the melt-bounded log-normal distribution of part II (Fig. II13). As reminder, using this probability law, the resistance values cannot outstrip $105 \mathrm{~m} \Omega$ which corresponds to a 1 $\mathrm{mm}$ thickness plate conductivity $\sigma=9524 \mathrm{~S} . \mathrm{m}^{-1}$. As a consequence, to ensure a good shielding efficiency with low current losses, the ES conductivity $\sigma_{E S}$ of the hybrid model is $\sigma_{E S}=\frac{\sigma}{100}=95.24{\mathrm{~S} . \mathrm{m}^{-1}}^{-1}$ for a $1 \mathrm{~mm}$ plate. In this paper, we focus on measurement on the ribs of Fig. 14. For each lightning shot, only one measurement with the Pearson probe is realized on one of these ribs due to a lack of instrumentation. In modeling, the current flowing on each rib is calculated. Indeed, these currents are computed doing the sum of the 
TABLE I

FUEL TANK ELEMENTS CHARACTERISTICS

\begin{tabular}{cccc}
\hline \hline & Material & Thickness $(\mathrm{mm})$ & Surface impedance $\left(\Omega \mathrm{m}^{-2}\right)$ \\
\hline Wedge & Aluminum & 5 & $8.33 \cdot 10^{-6}$ \\
Rib & Aluminum & 2 & $2.08 \cdot 10^{-5}$ \\
Spar & Composite 2 & 4 & $4.55 \cdot 10^{-2}$ \\
Panel & Composite 1 & 5 & $1.92 \cdot 10^{-3}$ \\
Panel + ECF195 & Composite 1 + ECF195 & 5 & $5.48 \cdot 10^{-4}$ \\
Panel + ECF815 & Composite 1 + ECF815 & 5 & $1.68 \cdot 10^{-4}$ \\
\hline \hline
\end{tabular}

TABLE II

CONDUCTIVITIES LINKED TO THE FUEL TANK MATERIALS

\begin{tabular}{lccccc}
\hline \hline & Aluminum & Composite 1 & Composite 1+ ECF195 & Composite 1 + ECF815 & Composite 2 \\
\hline Conductivity $\left(\mathrm{S} . \mathrm{m}^{-1}\right)$ & $2.400 \cdot 10^{7}$ & $1.040 \cdot 10^{5}$ & $3.647 \cdot 10^{5}$ & $1.194 \cdot 10^{6}$ & $5.500 \cdot 10^{3}$ \\
\hline \hline
\end{tabular}

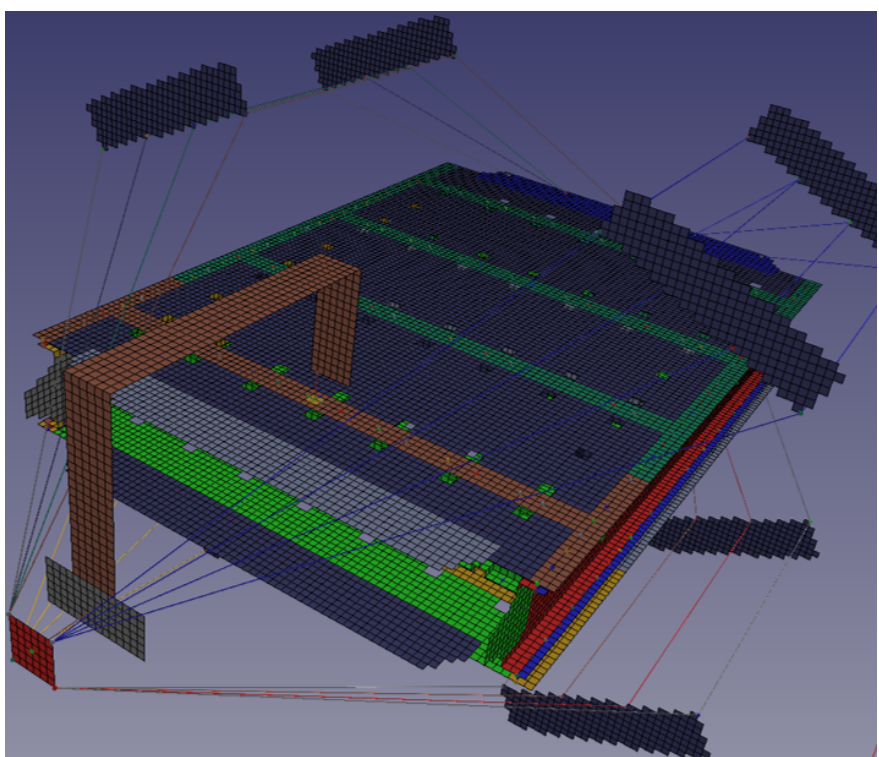

Fig. 13. Fuel tank FDTD mesh in an attachment configuration.

collected currents on the FDTD pink cell edges of Fig. 14. Furthermore, we note that these ribs are the closer the lightning injection.

The lightning injection is made on a fastener which ensure the ECF815/panel/rib link. This fastener is located between the rib R1 and L1 of Fig. 14. A specific fastener model is applied for this fastener. A star connection schema represents the fastener as illustrated in Fig. 15. The lightning injection is realized with a wire on the star point. Hence, the current can flow through four wires connected to the panel with ECF815 or through one wire connected to the rib. This specific fastener model is not constituted with lumped resistances. An ES is added on the involved aperture with the same conductivity as the hybrid model.

For the FDTD computation, a Gaussian waveform has been chosen rather than the double-exponential. Actually, the Gaussian waveform allows a faster convergence in the FDTD method. Moreover, we split the FDTD computation into two simulations with different frequency bands to save time. The

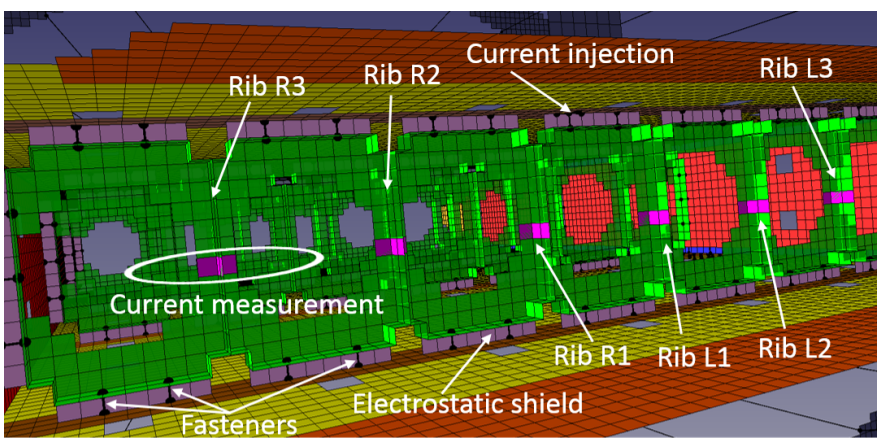

Fig. 14. Zoom on the measurement area.

first one from $100 \mathrm{~Hz}$ to $100 \mathrm{kHZ}$ until $1 \mathrm{~ms}$ is performed. The second one covers the $10 \mathrm{kHz}-1 \mathrm{MHz}$ bandwidth. For both of them, a low frequency acceleration technique is used, as in part I, in order to have reasonable computational time [14].

As in the previous parts, several simulations are performed. The aim is to surround the measured current taking into account the contact resistances uncertainties. In this paper, the simulation number is limited to fifty. Each simulation has a particular set of lumped resistances values following the melt-bounded log-normal distribution. Due to the high number of simulations, the CALI supercomputer of the University of Limoges is used.

\section{RESUlTS}

\section{A. Post-treatment process}

The current measurements are in the time domain until $1 \mathrm{~ms}$ with specific lightning injections presented in Fig. 10. With the FDTD method, the currents are computed in the time domain with a Gaussian waveform as source. Nevertheless, we choose to collect the simulated currents in the frequency domain and then to normalize them according to the Gaussian source. As a consequence, a post-treatment has to be made on these simulated currents in order to compare them with measurements. First, a filter is used for each simulated current in the overlapping bandwidth of both simulations (between 30 


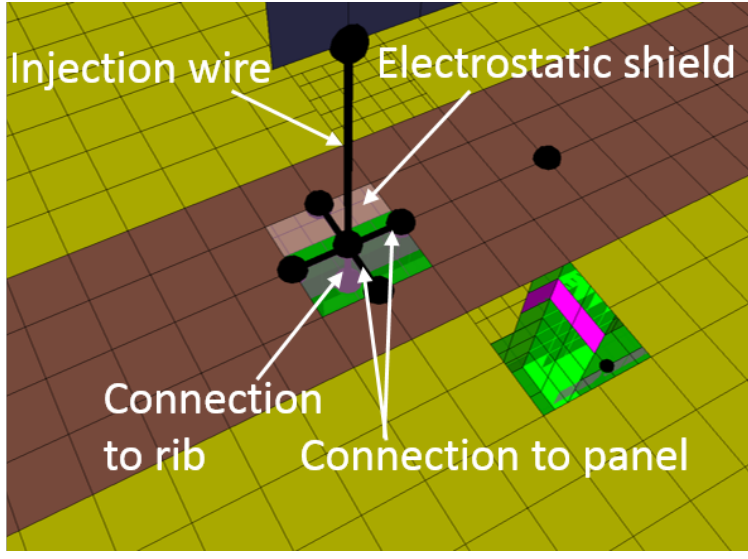

Fig. 15. Zoom on the lightning injection area. Star fastener model.

and $50 \mathrm{kHz}$ ) in the frequency domain. This operation allows reconstructing the signal $S_{\text {normalized }}^{\text {simulation }}(f)$ without abrupt break as in Fig. 7 at $100 \mathrm{kHz}$. Furthermore, a Fourier transform is applied on the measured injected current $S_{\text {source }}^{\text {measure }}(t)$ of Fig. 10. Hence, the resulting signal $S_{\text {source }}^{\text {measure }}(f)$ is multiplied by $S_{\text {normalized }}^{\text {simulation }}(f)$ to derive:

$$
S(f)=S_{\text {source }}^{\text {measure }}(f) \times S_{\text {normalized }}^{\text {simulation }}(f),
$$

with $S(f)$ the simulated current signal with the measured lightning source in the frequency domain. Finally, applying an inverse Fourier transform on $S(f)$, we obtain the expected $S(t)$ signal for the comparison with measurement in the time domain.

This process is used for all the following results in this paper.

\section{B. First results analysis}

In this sub-section, some results are presented and discussed. The proposed modeling is sufficient for the rib R3. Indeed, Fig. 16 shows that the measurement is well surrounded

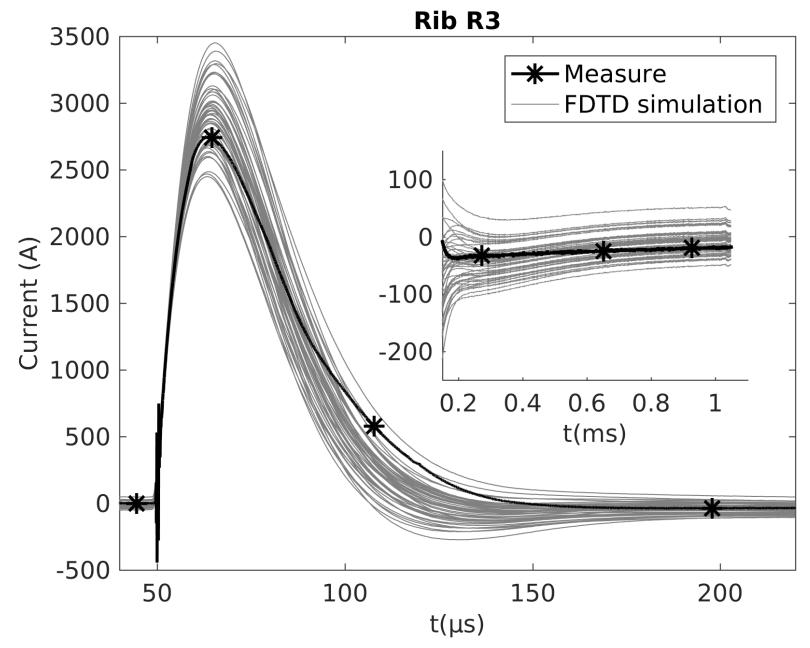

Fig. 16. Measurement (black line with asterisk) surrounded by the 50 simulated currents (grey lines) for the rib R3. The inset shows the surrounding from $0.2 \mathrm{~ms}$ until $1 \mathrm{~ms}$. by the 50 simulated currents in the short times as well as in the long times. The rise time, the pulse length and the amplitude of the measurement are in agreement with the proposed surrounding. Nevertheless, this modeling understates strongly the current flowing in the nearest ribs to injection as illustrated in Fig. 17. This substantial inaccuracy which concerns especially the short times seems to be due to a modeling failure. In particular, the star model as attachment fastener model is questioned in the next subsection.

\section{Sensitivity analysis}

The previous results presented in Fig. 17 suggest that too much current is flowing in the panel against the one flowing in the rib with the proposed modeling. To solve this issue different attachment fastener models are tested with the same random set of resistance following the log-normal law. Hence, a resistive star schema is built with the path to panel one hundred more resistive than the path to rib. Moreover, we experiment the extreme case noted direct schema without link to panel.
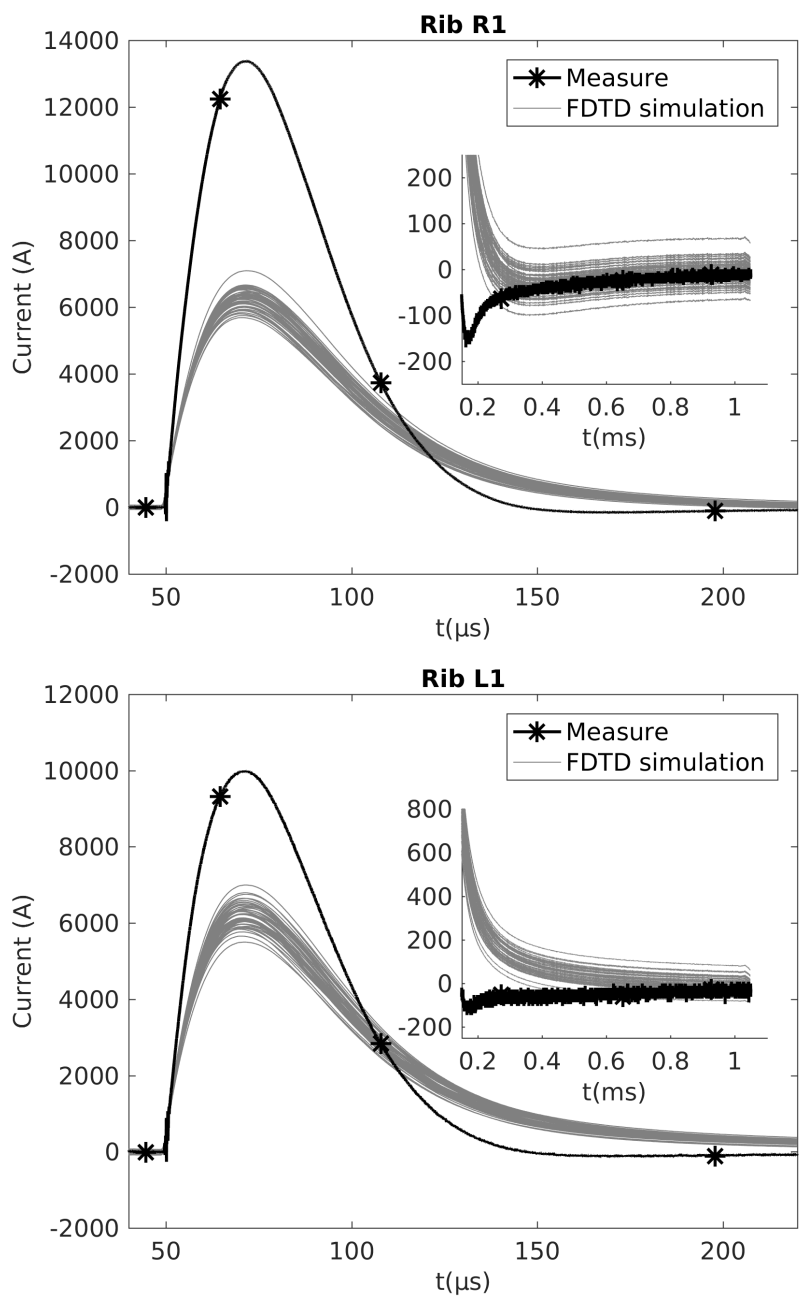

Fig. 17. Measurement (black line with asterisk) surrounded by the 50 simulated currents (grey lines) for the ribs R1 (at top) and L1 (at bottom). The inset shows the surrounding from $0.2 \mathrm{~ms}$ until $1 \mathrm{~ms}$. 

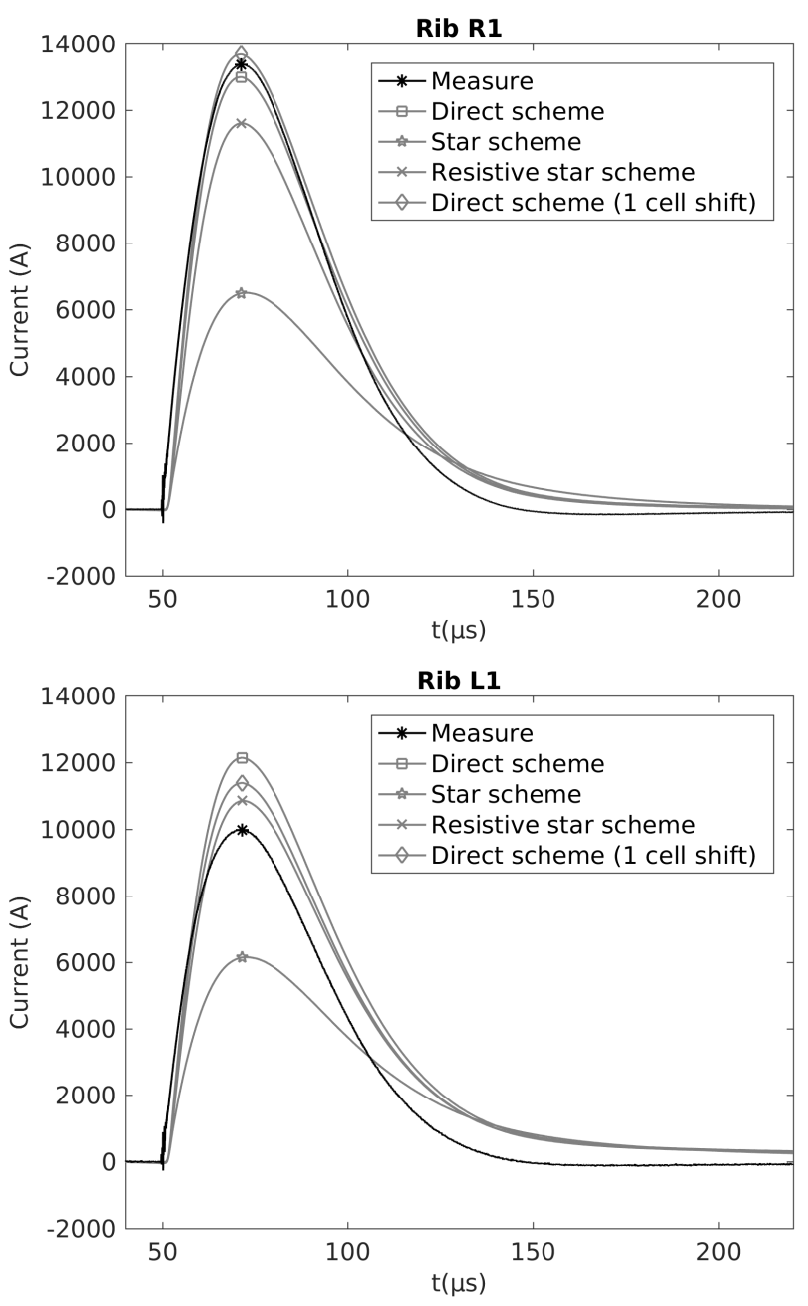

Fig. 18. Comparison between measurement and several modelings for the ribs R1 (at top) and L1 (at bottom).

Fig. 18 presents the computed currents of the different modelings for the rib closer to lightning injection in comparison with measurements. The previous assumption is validated; the current has to flow mainly in the rib. But even with the direct scheme, the current flowing in Rib L1 could be underestimated and the one in L1 overstated. Therefore, another source of modeling error which is the fastener position is analyzed. Indeed, the position of the fastener models in the FDTD modeling is not in perfect agreement with the one in measurement. Thus, the attachment fastener model is moved a one cell closer to the rib R1. The results presented in Fig. 18 are sufficient with an increase of current in the rib $\mathrm{R} 1$ and a drop in the rib L1 in contrast to the direct schema. This model seems to be the better one among the investigated ones.

\section{Overall results discussion}

According to the previous subsection, a star scheme could be inadequate for the attachment fastener model even with resistive paths. Direct scheme enhances clearly the agreement with measurement for the ribs closer to lightning attachment. As for the star scheme, 50 simulations are performed with the both direct schema. The 50 random sets of contact resistances are the same for the three different modelings. Table III summarizes the surrounding performances of each modeling. These performances are studied from three criteria (rise time, amplitude, pulse length). These results highlight that several modeling inaccuracies lead to a wrong estimation. Among other things, particular attention has to be given to the attachment fastener modeling. A direct scheme should be the better modeling and thus, it involves that the whole current flows in the rib. Hence, the use of ECF for the panel protection has to be questioned. Furthermore, the current estimation closer to the lightning injection is sensitive to the fastener mapping in the FDTD grid. The proposed direct scheme with one cell shift is efficient for the rise time, amplitude and pulse length assessment. Nevertheless, this modeling is not able to surround the measurement of R1 and L1 in all the time range as illustrated in Fig. 19. Indeed, the signal attenuation is faster in measurement. Thus, we investigate another probable source of error which is the probability law of the uncertainty model. Hence, the results of 50 simulations with three different probability laws (log-normal, Gaussian and uniform) are compared. The log-normal law is still the melt-bounded law as in the previous subsections. The Gaussian law is centered at $27 \mathrm{~m} \Omega$ with a $10 \mathrm{~m} \Omega$ standard deviation, ie with the same mean as the log-normal law and a smaller standard deviation. The uniform law is bounded from $0.01 \mathrm{~m} \Omega$ from $105 \mathrm{~m} \Omega$ and hence with higher mean and standard deviation than the log-normal law ones. The probability density function of the 12750 generated samples (255 fasteners x 50 simulations) of each law is illustrated in Fig. 20. The upper and lower currents of each uncertainty model are shown in Fig. 21. Moreover, Fig. 21 presents the mean of the 50 simulated currents at three different times with the associated standard deviation for each uncertainty model. These indicators depict the density of the 50 simulated currents for each uncertainty model. Obviously, the broadest surrounding is the one using the uniform law. Indeed, we test all the possible combinations with this kind of uncertainty model. It allows a surrounding minimizing the risk of wrong prediction. This kind of law is not enough accurate for a sufficient prediction. Moreover, the surrounding is broader with the Gaussian law than with the log-normal law even if the Gaussian law standard deviation $(10 \mathrm{~m} \Omega)$ is smaller than the log-normal law one $(17 \mathrm{~m} \Omega)$. This result validates the log-normal law as the more accurate uncertainty model especially since the standard deviation of the simulated current is smaller using the log-normal law. Furthermore, looking at the signal attenuation, the simulated curves are almost parallel. Therefore, the inaccuracy on the rib L1 is not due to the choice of the uncertainty model. An accurate mapping of each fastener could likely enhanced the surrounding. In another hand, the Pearson probe used for measurement could be questioned, especially for measurement with low current amplitude. A wrong estimation of the fuel tank material conductivities (in particular for composite elements) could also lead to the error on the waveform assessment. Otherwise, the authors claims that all the other measurements (in the others ribs) are perfectly surrounded by simulations using the lognormal law as uncertainty model. 
TABLE III

SURROUNDING PERFORMANCE RESULTS ON THREE CRITERIA FOR SEVERAL MODELINGS

\begin{tabular}{cccccccccc}
\hline \hline & \multicolumn{3}{c}{ Star scheme } & \multicolumn{3}{c}{ Direct schema } & \multicolumn{3}{c}{ Direct scheme (1 cell shift) } \\
\hline & Rise time & Amplitude & Pulse length & Rise time & Amplitude & Pulse length & Rise time & Amplitude & Pulse length \\
\hline R3 & $\checkmark$ & $\checkmark$ & $\checkmark$ & $\checkmark$ & $\checkmark$ & $\checkmark$ & $\checkmark$ & $\checkmark$ & $\checkmark$ \\
R2 & $\checkmark$ & $x^{-}$ & $x^{-}$ & $\checkmark$ & $\checkmark$ & $\checkmark$ & $\checkmark$ & $\checkmark$ \\
R1 & $\checkmark$ & $x^{-}$ & $x^{+}$ & $\checkmark$ & $x^{-}$ & $x^{+}$ & $\checkmark$ & $\checkmark$ & $\checkmark$ \\
L1 & $\checkmark$ & $x^{-}$ & $x^{+}$ & $\checkmark$ & $x^{+}$ & $x^{+}$ & $\checkmark$ & $\checkmark$ & $\checkmark$ \\
L2 & $\checkmark$ & $\checkmark$ & $\checkmark$ & $\checkmark$ & $\checkmark$ & $\checkmark$ & $\checkmark$ & $\checkmark$ \\
R3 & $\checkmark$ & $\checkmark$ & $\checkmark$ & $\checkmark$ & $\checkmark$ & $\checkmark$ & $\checkmark$ & $\checkmark$ & $\checkmark$ \\
\hline \hline
\end{tabular}

$\checkmark$ indicates that simulations surround measurement

$x^{-}$indicates that the simulation surrounding understates measurement

$\mathrm{x}^{+}$indicates that the simulation surrounding overstates measurement

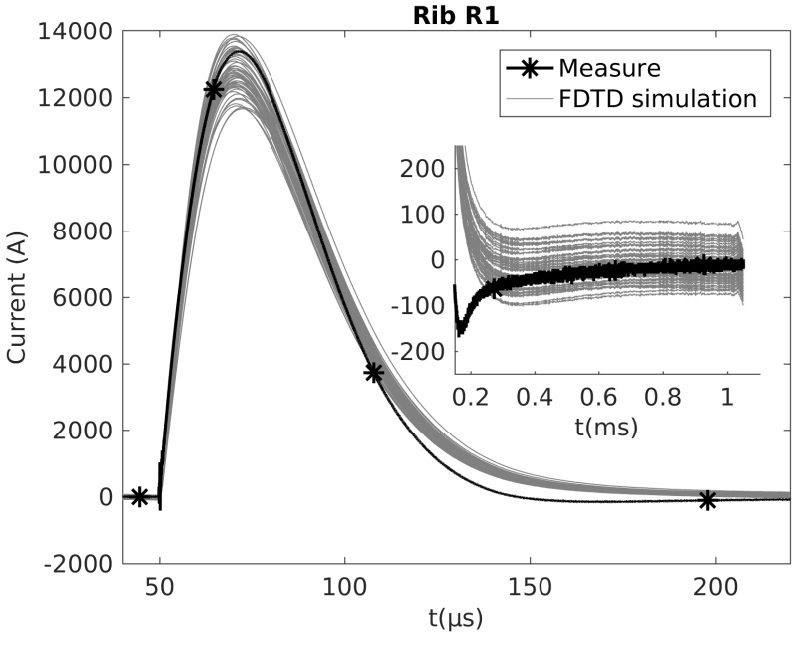

Rib L1

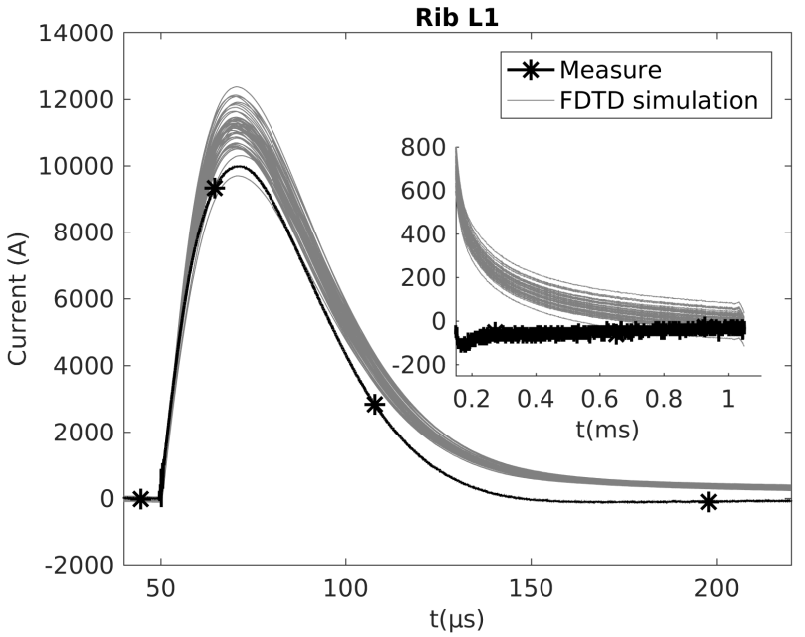

Fig. 19. Measurement (black line with asterisk) surrounded by the 50 simulated currents (grey lines) with the direct scheme (with one cell shift) for the ribs R1 (at top) and L1 (at bottom). The inset shows the surrounding from $0.2 \mathrm{~ms}$ until $1 \mathrm{~ms}$.

\section{CONCLUSION}

This paper validates the proposed approach in Part I for the fastener lightning current assessment in aircraft fuel tank with the FDTD method. The fastener model of Part I is enhanced in order to provide a good shielding efficiency in the generic fuel tank used in the two previous parts. This enhancement is performed adding an electrostatic shield on

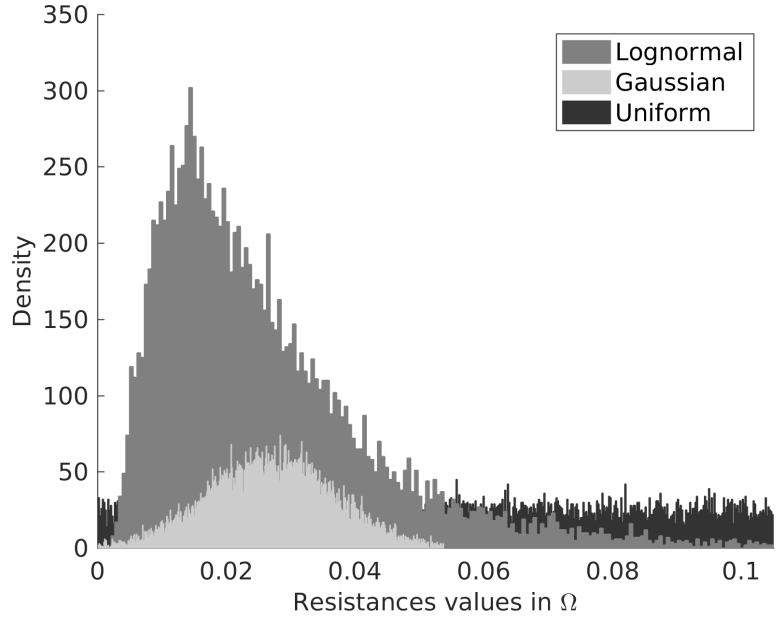

Fig. 20. Probability density histograms of computed data

the aperture which is made in the FDTD grid by the fastener model implementation. The conductivity of this electrostatic shield which has to be applied is widely discussed. We note that a very low conductivity (around few hundred of siemens per meter) is sufficient to ensure a good shielding efficiency in the lightning frequency bandwidth. Then, this new model called hybrid model is implemented in a FDTD modeling of a realistic fuel tank. The main parameter of the hybrid model is a resistor which represents the equivalent contact resistance of fastener. Therefore, this resistive parameter takes a random value according to the improved uncertainty model of part II which is a melt-bounded log-normal law. As in the previous parts, several simulations are performed in order to take into account the uncertainties. In comparison with measurement, we note that a specific attention has to be given to the fastener where the lightning injection is performed. This fastener ensures the link between a composite panel with an extended copper foil and an aluminum rib of the fuel tank. A sensitivity analysis highlights that the modeling of this fastener has a strong incidence on the current assessment close to the injection. In particular, we conclude that all the current is flowing in the rib which puts into question the relevance of the extended copper foil as protection. Several simulations are performed with a model taking into account this conclusion. The results highlights 1) the significant sensitivity of current 

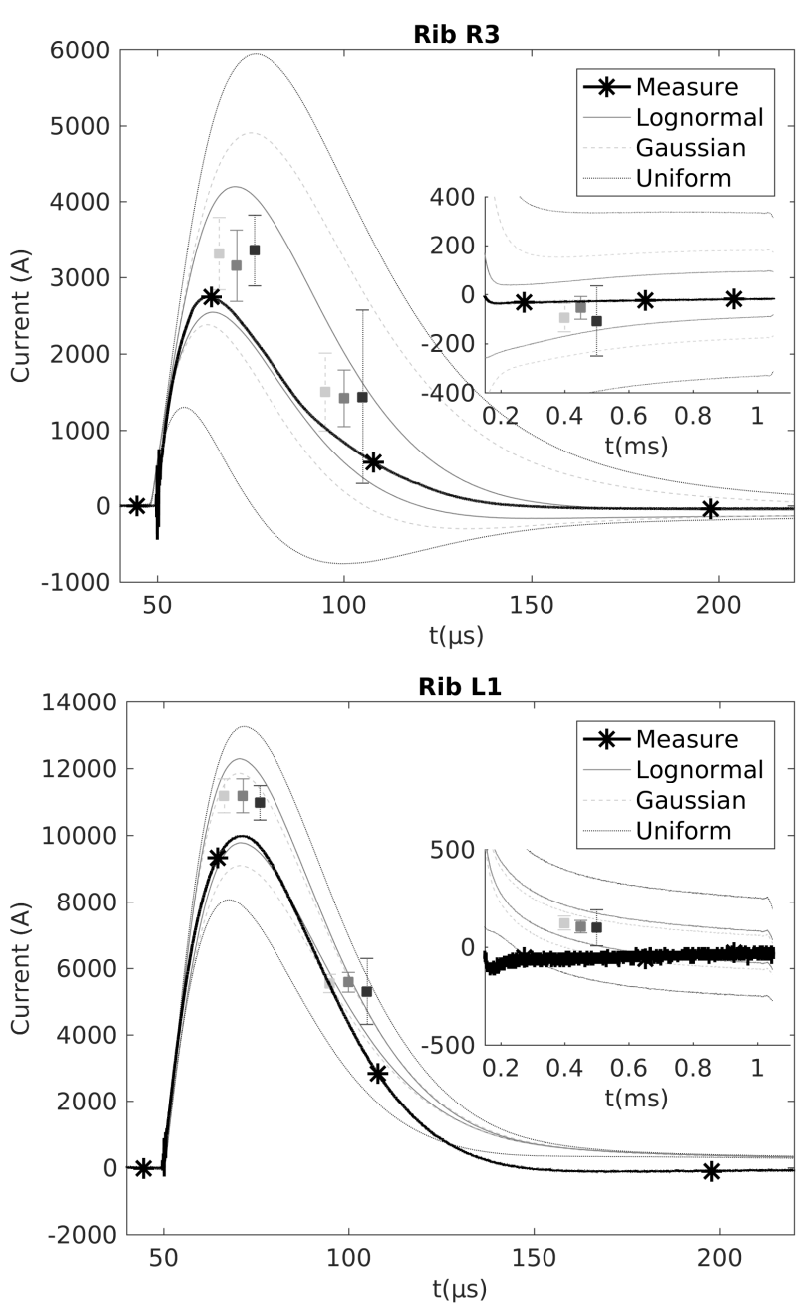

Fig. 21. Upper and lower bounds of the 50 simulated currents with a lognormal, Gaussian and uniform uncertainty model for the ribs R3 (at top) and L1 (at bottom). The error bars are centered in the empirical mean curve of each uncertainty model at $71 \mu \mathrm{s}, 100 \mu \mathrm{s}$ and $450 \mu \mathrm{s}$. They represent the empirical standard deviation of the 50 simulations for each uncertainty model. The inset shows the surrounding from $0.2 \mathrm{~ms}$ until $1 \mathrm{~ms}$.

distribution to the contact resistances and 2) a good agreement with measurement which validates the proposed approach. Moreover, still close to the lightning injection, we note that the current assessment is sensitive to the fastener mapping in the FDTD grid. A perfect knowledge of this mapping is required for an accurate assessment. Furthermore, a comparison study has shown that the melt-bounded log-normal law is reliable as uncertainty model. In the future, it is planned to investigate the modeling inaccuracies (fastener mapping, fastener modeling, etc...) to improve the current assessment. Moreover, methods which avoid to perform several full-wave simulations will be studied in order to take into account the contact resistance uncertainties with a faster method.

\section{REFERENCES}

[1] L. Chemartin, P. Lalande, and F. Tristant, "Modeling and simulation of sparking in fastening assemblies." in ICOLSE 2013, Seattle, United States, Sep. 2013. [Online]. Available: https://hal-onera. archives-ouvertes.fr/hal-01058554
[2] F. Fustin, F. Tristant, J.-P. Moreau, and F. Terrade, "Fuel tank safety 3D distributions on fasteners/assemblies outside and inside fuel tanks," in ICOLSE 2013, Seattle, United States, Sep. 2013.

[3] I. Revel, G. Peres, B. Lepetit, and F. Flourens, "Understanding of sparking phenomenon in CFRP assemblies," in International Conference on Lightning and Static Electricity, Paris, France, 2007.

[4] P. Monferran, C. Guiffaut, A. Reineix, F. Fustin, and F. Tristant, "Fastening assemblies modelling in finite difference time domain," in 2018 International Symposium on Electromagnetic Compatibility (EMC EUROPE), Aug 2018, pp. 521-526.

[5] H. Mulazimoglu and L. Haylock, "Recent developments in techniques to minimize lightning current arcing between fasteners and composite structure," in Proceedings Int. Conf. Lightning and Static Electricity, Oxford,UK, 2011.

[6] P. Monferran, C. Guiffaut, A. Reineix, F. Fustin, and F. Tristant, "Lightning effects on fastening assemblies of an aircraft fuel tank, part I: Uncertainties assessment with statistical approach."

[7] _ _ "Lightning effects on fastening assemblies of an aircraft fuel tank, part II: FDTD modeling merged with a circuit model supplemented by a statistical model."

[8] J. P. Bérenger, "Plaques minces aux différences finies," in Gème Colloq. Int. Expos. Compat. Electromagn.(CEM), 1992, pp. 298-303.

[9] R. J. Luebbers and K. Kunz, "FDTD modeling of thin impedance sheets (radar cross section calculation)," IEEE Transactions on Antennas and Propagation, vol. 40, no. 3, pp. 349-351, 1992.

[10] C. Railton and J. P. McGeehan, "An analysis of microstrip with rectangular and trapezoidal conductor cross sections," IEEE Transactions on Microwave Theory and Techniques, vol. 38, no. 8, pp. 1017-1022, 1990

[11] J. P. Bérenger, "A perfectly matched layer for the absorption of electromagnetic waves," Journal of computational physics, vol. 114, no. 2, pp. 185-200, 1994.

[12] _ - "Perfectly matched layer for the fdtd solution of wave-structure interaction problems," IEEE Transactions on Antennas and Propagation, vol. 44, no. 1, pp. 110-117, Jan 1996.

[13] — - "Perfectly matched layer (PML) for computational electromagnetics," Synthesis Lectures on Computational Electromagnetics, vol. 2, no. 1, pp. 1-117, 2007.

[14] R. Holland, "Finite-difference time-domain (FDTD) analysis of magnetic diffusion," IEEE Transactions on Electromagnetic Compatibility, vol. 36, no. 1, pp. 32-39, Feb 1994. 